\title{
Heroum virtutes: Intessere eroi, intessere tele, intessere chiose, "artigiani” del mondo antico
}

\author{
Marilena Caciorgna \\ Università di Siena \\ caciorgna@unisi.it
}

\section{Riassunto}

Le fil rouge di questo breve saggio è l'arte del "tessere" che lega gli antichi ai "moderni": dall'èkphrasis di Catullo intessuta nella coperta nuziale alla spalliera di Girolamo del Pacchia con l'abbandono di Arianna fino alle eroine intrecciate da artisti del Rinascimento, da Penelope che tesse il sudario di Laerte a Minerva maestra nell'arte della tessitura ai commentatori dei testi antichi, umanisti-artigiani che intessono chiose, contaminano fonti e creano nuovi "tessuti narrativi", come nel caso delle spalliere de Musée du Petit Palais di Avignone raffiguranti Storie di Minosse, Pasifae, Teseo, Arianna e Fedra. Letterati spesso dimenticati, "artigiani del mondo antico", chiosatori anonimi, ma che hanno influito sull'iconografia dei dipinti del Quattro-Cinquecento sia attraverso le loro postille che servono a intessere il ductus narrativo sia come ideatori del programma consultati da committenti prestigiosi e attentamente seguiti da artisti in cerca di ispirazione.

Parole chiave: tessere; commentatori di testi antichi; umanisti-artigiani; Arianna; Penelope; Giovanni delle Bande Nere; Catullo; Ovidio; Bartolomeo Merula; Raffaele Regio; Girolamo del Pacchia; Domenico Beccafumi; spalliere Campana.

Abstract. Heroum virtutes: weaving heroes, weaving canvases, weaving glosses, "artisans"
of the ancient world

The guiding thread of this short essay is the art of "weaving" that binds the ancients to the "moderns": from Catullus' èphrasis woven into the wedding blanket to the chair back by Girolamo del Pacchia with the abandonment of Ariadne up to the weaving heroines by artists of the Renaissance, from Penelope who weaves the shroud of Laertes to Minerva, teacher of the art of weaving, to the commentators of ancient texts, humanist-artisans who weave glosses, blend together sources and create new "narrative fabrics", as in the case of the chair backs now in the Musée du Petit Palais in Avignon depicting stories of Minos, Pasiphae, Theseus, Ariadne and Phaedra. Often forgotten writers, "artisans of the ancient world", anonymous commentators who influenced the iconography of paintings of the fifteenth and sixteenth centuries both through their annotations that serve to weave the narrative ductus and as creators of the iconographical programs, consulted by prestigious patrons and attentively followed by artists looking for inspiration.

Keywords: weaving; commentators of ancient texts; humanist-artisans; Ariadne; Penelope; Giovanni delle Bande Nere; Catullus; Ovidius; Bartholomaeus Merula; Raphael Regius; Girolamo del Pacchia; Domenico Beccafumi; spalliere Campana. 
Il carme 64 di Catullo è un epitalamio, un componimento poetico scritto e recitato in occasione di nozze, che descrive l'unione di Peleo, che aveva partecipato alla spedizione degli Argonauti, con Teti, la più bella fra le Nereidi. Il tessuto narrativo si interrompe con una lunga digressione, una èkphrasis contenente la rappresentazione di vicende mitiche ricamate nella coltre nuziale istoriata degli sposi, che copre il letto al centro della sala, sedibus in mediis, adornato con fregi d'avorio indiano. ${ }^{1}$ La coperta "parlante" è istoriata mira arte, con arte stupenda, e rappresenta heroum virtutes, le imprese virtuose degli eroi antichi, exempla per gli sposi novelli:

La coperta, istoriata con fatti d'uomini antichi, racconta con arte stupenda imprese d'eroi. ${ }^{2}$

La descrizione della coltre permette al poeta di ricordare il risveglio di Arianna abbandonata da Teseo nell'isola di Dia e l'arrivo di Bacco col chiassoso corteo di Menadi e Satiri. Il poeta si sofferma in particolare sul penoso lamento dell'eroina impietrita per il dolore, essendosi scoperta abbandonata sul lido deserto, e su di una serie di riferimenti agli antecedenti della storia.

Gli antichi raffiguravano gli amori degli dei nelle coltri nuziali, ed anche nel XV secolo si diffonde l'uso di intessere le gesta degli eroi negli arazzi e nei tessili che avrebbero impreziosito nobili dimore del XV secolo. La loro funzione non è tuttavia soltanto estetica, ma anche, attraverso il soggetto rappresentato, moralizzante. Tale abitudine è largamente attestata dai fondi documentari e da alcuni testimoni. A Siena, Maestro Giachetto, valente arazziere d'Arras, viene incaricato, il 30 aprile 1445, della "fattura di una spalliera che doveva sostituire il panno che fino allora era stato nella sala in cui si adunavano i Priori: l'arazzo doveva essere delle dimensioni del panno che avrebbe sostituito e dovevano esservi raffigurati dieci personaggi in piedi, divisi fra loro da alberi e con gli altri ornamenti consueti in simili lavori, e il tutto in ottima lana". ${ }^{3}$ Dalla descrizione si evince che la rappresentazione si ispira al canone degli Uomini Famosi, serie di personaggi illustri del mondo antico a figura intera stante.

Fra i testimoni si veda il brano tessile con la rappresentazione di Penelope eseguito per il cardinal Ferry de Clugny, vescovo di Tournai verso il 1480, frammento di un arazzo smembrato raffigurante una serie di Donne Famose (Fig. 1). ${ }^{4}$ Sotto la figura, intenta a tessere il sudario di Laerte, si legge la scritta "PENELOPE CÔIVNX SÊPER VLIXIS ERO" (sarò sempre Penelope, la moglie di Ulisse) che deriva dalle Heroides di Ovidio $(1,84)$, al fine di enfatizzare la fedeltà dell'eroina e la sua capacità di attendere l'amato lontano. $\mathrm{Nel}$

1. Pulvinar vero divae geniale locatur / sedibus in mediis, Indo quod dente politum / tincta tegit roseo conchyli purpura fuco (vv. 47-49).

2. Haec vestis priscis hominum variata figuris / heroum mira virtutes indicat arte (vv. 50-51). Trad. G. Chiarini, in Catullo, ed. 1996, p. 153.

3. Cecchini, 1962 , p. 57.

4. Il frammento è conservato a Boston, Museum of Fine Arts. Cfr. in proposito Sanfilippo, 2013, p. 138. 
Quattrocento, le Eroidi, una serie di lettere che il poeta immagina scritte da eroine ai loro amanti o mariti lontani e, in tre casi, da eroi cui segue la risposta delle rispettive amate, grazie anche alle traduzioni e volgarizzamenti, diventano un testo alla moda, come è dimostrato dalla loro frequente presenza nelle biblioteche, e destinato a un pubblico prevalentemente femminile. ${ }^{5}$ In un codice del primo quarto del Quattrocento di provenienza fiorentina, l'Ambrosiano S.P.13bis, decorato con numerose miniature attribuite al Maestro del Codice Squarcialupi, a carta III $v$, è raffigurato, a piena pagina, all'interno di una cornice prospettica, Ovidio. Il poeta indossa un'ampia toga e mostra con la mano sinistra un libro aperto dove si legge "Ovidio DELLE DONNE POE (sic)", ${ }^{6}$ titolo piuttosto evocativo, che si apre a interpretazioni diverse di notevole suggestione per gli studi della Rezeption. Una convergenza significativa risulta infatti dalla produzione di cassoni, spalliere, deschi da parto del XV secolo raffiguranti episodi di eroi e, in particolare, eroine dell'antichità, quali exempla virtutis, offerti all'imitazione dello spettatore. ${ }^{7}$ Il carattere esemplare dei volgarizzamenti delle Heroides, d'altronde, è dimostrato dal contenuto dei prologhi anteposti dai traduttori alle epistole in cui si presentano al lettore una serie di esempi "d'amore" sequenda aut vitanda (da seguire o da evitare).

Per gli studi sulla ricezione del mondo antico è interessante rilevare dunque come le Heroides, tra Medioevo e Rinascimento, attraverso volgarizzamenti, traduzioni e testi introduttivi alle epistole, diventino una sorta di repertorio mitologico a carattere moralizzante in cui si esplicano ad esempio le conseguenze di un abbandono dell'amata da parte dell'eroe, un monito per quei mariti che abbiano deciso di compiere questo gesto con le proprie mogli. D'altra parte le Heroides avevano costituito in origine l'invenzione di un nuovo genere letterario sorto da due tradizioni diverse quali l'elegia e la tradizione epico-tragica.

Ancora la figura di Penelope intenta a tessere la tela in un telaio verticale compare al centro di una formella ricamata in un paliotto del XVI secolo che si conserva a Pienza (Fabbriceria del Duomo) ampiamente rimaneggiato: "Questo ricamo... è straordinariamente giunto fino a noi per un felice errore che ha identificato per secoli l'immagine come una Santa Cecilia, collocandola al centro di un apparato sacro e preservandola così dalla distruzione e dalla noncuranza a cui sono andate invece incontro altre opere di soggetto profano". 8 Alla Penelope pientina si connette una testimonianza d'archivio di recente rinvenuta che permette di implementare il modulo delle eroine all'interno del corpus di tessili. Nel tardo XVI secolo si trovavano nella sagrestia del Duomo di Siena due guanciali con ricami raffiguranti Lucrezia e Giuditta: "Un paio

5. Bec, 1984, passim. Zaggia, Ceriana 1996, pp. 7-8.

6. Zaggia, Ceriana 1996, pp. 8, 23-39.

7. Sulla fortuna delle Heroides nella tradizione e nell'iconografia del Rinascimento si veda Caciorgna 2004; Caciorgna 2008; Caciorgna 2015.

8. Bari, 2017, p. 42. 
di guanciali di raso cremisi raccamati d'oro e perle con due figure e festone in mezzo, nell'uno è Giuditta e nell'altro è Lucrezia".?

Non rappresenta invece Penelope un dipinto di Domenico Beccafumi conservato al Seminario Patriarcale di Venezia (Fig. 2), da alcuni studiosi connesso alla serie 'domestica' per la camera di Francesco Petrucci eseguita intorno al 1519 , in cui si prospetta un'imponente figura femminile interpretata fino a oggi come la moglie di Ulisse, ma che dunque pare da doversi riconoscere in Minerva. ${ }^{10}$ La dea infatti, definita textrinae artis magistra, oltre a essere una divinità guerriera e simbolo di Sapienza, presiede alle attività domestiche come la filatura e la tessitura. ${ }^{11}$ Ovidio (Fasti 3, 817-20) spiega come le fanciulle, ottenuto il favore di Pallade, possano apprendere a cardare la lana e a scorrere con la navetta fra l'ordito ben fitto, e a spingere con il pettine i fili troppo radi. 12

Al di là delle fonti antiche la connotazione della dea quale maestra dell'arte della tessitura ricorre anche nei testi medievali, fra i quali si segnala il $D e$ mulieribus claris di Boccaccio per il forte influsso esercitato nell'iconografia del Rinascimento: "In secondo luogo, vogliono che abbia inventato l'arte della lana, fino ad allora a tutti ignota. Ella insegnò infatti il metodo di purificare la lana, di ammorbidirla con pettini di ferro, di porla sulla rocca e di filarla colle dita. Così Minerva inventò l'arte della tessitura, insegnando come i fili dovessero essere intrecciati e raddensati col pettine e come il tessuto dovesse essere rassodato calcandolo col piede. In lode di questo suo lavoro si cita la famosa gara con Aracne di Colofone". ${ }^{13}$

Infine, fra le fonti tardo antiche, si segnala Sidonio Apollinare, autore del carme XV, poema dedicato alle nozze di Polemio e Araneola, Pallade pronuba: "nella seconda parte vengono celebrate le laudes dei due promessi sposi, rilevandone le specchiate qualità e la nobile origine: Polemio sarà celebrato come filosofo, Araneola come abilissima tessitrice. La presenza di Atena nel carme non è occasionale, ma organicamente collegata alle laudes dei due sposi perché personalmente responsabile dei due campi di attività in cui essi eccellono". ${ }^{14}$ Nel carme (vv. 126-131) è ricordato il laboratorio di Minerva in cui si tesse il manto di Giove, tinteggiato con la porpora Sidonia: "Ma altrove, nel laboratorio di Minerva, in primo piano brilla il manto di Giove, i cui serici fili tingeva la porpora Sidonia, due volte bollita nella caldaia. Sebbene impregnato, il tessuto non alita solo la porpora, perché ha un lampo intessuto, e nel filo rigido ardeva la porpora appesantita da un frammento di folgore”. ${ }^{15}$

9. Bari, 2017, p. 43. Cfr. AOMS, Inventari, 1493 (868), c. $4 v$.

10. Caciorgna, 2013, pp. 113-115.

11. Firmicus Maternus, De errore profanarum religionum, 16, 1.

12. Cfr. anche Virgilio, Eneide VIII, 409; Tibullo II, 1, 61-66. Ovidio ricorda il legame tra Minerva e la tessitura anche in Metamorfosi IV, 32-36 nel racconto della vicenda mitica delle figlie di Minia.

13. Trad. it. V. Zaccaria, in G. Boccaccio, ed. 1967, p. 40.

14. Trad. it. G. Ravenna, in Sidonio Apollinare, ed. 1990, pp. 12-13.

15. Trad. it. G. Ravenna, in Sidonio Apollinare, ed. 1990, pp. 35. 
La solenne e fiera postura della donna dipinta da Beccafumi, il volto algido e il braccio che sembra impugnare un'asta invece di un telaio sembrano ispirarsi a qualche modello della statuaria classica. Rimanda all'antico anche il bassorilievo a destra del dipinto raffigurante forse Minerva che, in abiti più virili rispetto alla figura stante, insegna alle fanciulle l'arte della tessitura. La tela ordita dalla figura muliebre beccafumiana, inoltre, è purpurea come il manto di Giove mentre il sudario di Laerte, intessuto da Penelope, è di colore bianco. $^{16}$

Per tornare all'Epithalamium di Catullo che predilige la storia di Arianna per la coltre nuziale e alle Heroides di Ovidio e il loro influsso negli arredi domestici, rivolgiamo la nostra attenzione a una spalliera attribuita al pittore Girolamo del Pacchia che si conserva nella collezione di Palazzo Chigi Saracini raffigurante il distacco di Teseo da Arianna (Fig. 3). Nel carme catulliano la storia è intessuta nella coltre, nel caso della spalliera senese è dipinta nella tavola, parte dell'arredo di una camera da letto: la storia di Arianna è dunque utilizzata sia dagli antichi che dai "moderni" come tematica nuziale. Nella spalliera, inoltre, Arianna non è soltanto vittima di un destino infelice, ma anche la protagonista di un'unione a lieto fine, quella con Dioniso.

$\mathrm{Al}$ di là delle riprese da Catullo, una spia che tradisce, in alcuni dettagli, l'ispirazione diretta alle Heroides (Ariadna Theseo, vv. 41-44) è il candido velo appeso da Arianna a un ramo per farsi notare da Teseo che ha preso il largo con la sua nave. ${ }^{17}$ Per la fanciulla il viaggio finisce qui, l'amante è ormai troppo lontano. Questo elemento distintivo che connota la figura di Arianna si codifica in alcuni manoscritti di scuola francese preziosamente ornati, contenenti la traduzione delle Heroides di Octovien de Saint-Gelais, poeta e volgarizzatore della corte di Luisa di Savoia, nominato vescovo di Angoulême da Carlo VIII. ${ }^{18}$ In una delle splendide miniature che illustra la decima epistola ovidiana, il gesto di Arianna viene enfatizzato: non compare soltanto sullo sfondo, ma anche in primo piano quasi fosse un attributo che la contraddistingue. ${ }^{19}$ Con la mano destra agita il velo, candida velamina, applicato a un lungo bastone, longa virga. In questo caso la bandiera, legata all'asta, sembra divenire l'attributo che caratterizza l'infelice eroina. Nell'altra miniatura, la fanciulla, ridestatasi senza trovare al suo fianco l'amato, è disorientata e il suo equilibrio appare instabile. Si veda anche lo sfondo di una miniatura di Jean Pichore, verso il 1505-1510, esposta alla mostra France 1500 in cui Arianna sventola il velo a Teseo ormai partito. In primo piano l'eroina scrive l'epistola, mentre nella parte centrale è adagiata sul letto sotto una tenda (Fig. 4). ${ }^{20}$

16. Sanfilippo, 2013, p. 20.

17. Si non audires, ut saltem cernere posses, / iactatae late signa dedere manus; / candidaque inposui longae velamina virgae, / scilicet oblitos admonitura mei.

18. Parigi, Bibliothèque National de France, Richelieu Manuscrits Français 873 e 874 . Sulle miniature illustranti il volgarizzamento francese cfr. Caciorgna, 2008, pp. 37-70.

19. Parigi, Bibliothèque Nationale de France, Richelieu Manuscrits Français 874.

20. Paris, Bibliothèque de l'Assemblée nationale, Ms. 1466. Cfr. C. Zöhl, in France 1500 entre Moyen Age et Renaissance 2010, pp. 125-126. 
Se istituiamo un confronto con la miniatura, nella spalliera di Girolamo del Pacchia troviamo dunque l'immagine archetipica di Arianna che ostenta il velo, un dettaglio rivelatore, o formula del pathos, che riconduce anche al testo ovidiano. Nella tavola la sventurata eroina, ridestatasi da un profondo sonno, ha ormai subìto l'amara disillusione poiché scorge la nave dell'amato mentre scioglie le vele per prendere il vasto mare: appare raffigurata con il seno scoperto, su una riva paludosa dai toni lividi, cosparsa di una vegetazione caratteristica dei paesaggi lacustri, che accresce la desolazione del luogo e riflette lo stato d'animo della protagonista del dipinto. Leroina, pervasa da un sentimento di angoscia, accenna con la mano esausta, "stanca di colpire il triste petto", verso Teseo che rivolge uno sguardo estremo alla costa ormai remota. ${ }^{21}$ Anche il tendere le braccia verso l'amato, sembra richiamare l'epistola ovidiana, Has tibi plangendo lugubria pectora lassas, I infelix tendo trans freta longa manus (Le mie mani, stanche di colpire il triste petto, io sventurata tendo a te, al di là del vasto mare: Arianna a Teseo, 10,136-52).

Nel dipinto si riscontrano inoltre altri tratti ovidiani, con particolare riferimento all' Ars amatoria, come il dettaglio iconografico più suggestivo, le esili canne in primo piano, che riflettono lo stato d'animo di Arianna. La fragile eroina, dopo aver subito l'abbandono, trema per lo spavento, ut levis in madida canna palude tremit, "come la canna leggera nell'acqua della palude" $(1,552)$. Ancora all'Ars amatoria si ispira il particolare sullo sfondo, in alto, con il carro di Bacco che si orna di grappoli d'uva e viene trainato da tigri appaiate (1, $547-548) .{ }^{22}$

Il dettaglio distintivo ovidiano del velo bianco legato a una canna ci conduce ora all'analisi di quattro spalliere che si conservano nel Musée du Petit Palais di Avignone raffiguranti Storie di Minosse, Pasifae, Teseo, Arianna e Fedra (Figg. 5-8), provenienti dalla collezione di Giovanni Pietro Campana, appassionato cultore di archeologia e collezionista di 'primitivi'. ${ }^{23}$ In un celebre saggio pubblicato nel 1976, Federico Zeri individua nell'autore dei pannelli una congiunzione tra Firenze e la Francia, verso il 1510-15. Denominato "Maestro dei cassoni Campana" proprio per la provenienza dei pannelli di Avignone, Zeri ritiene che il pittore, per i caratteri nordici del suo stile, sia un maestro di origine francese presente a Firenze agli inizi del Cinquecento. ${ }^{24}$ In tempi recenti è stata confermata l'ascendenza francese dell'artista da parte di Christine Seidel, che riconduce lo stile del Maestro all'arte del sudest della Francia, in particolare al lionese e la pista che potrebbe condurre

21. Ovidio, Heroides X, 136-52.

22. Per un approfondimento: M. Caciorgna, s.v. Arianna, in Caciorgna, Guerrini 2003, pp. 33-34.

23. Laclotte, Moench 2005, pp. 130-131.

24. Zeri, 1976, pp. 75-87. Annamaria Bernacchioni (2011, pp-117-127) propone l'identificazione del maestro con 'Gallo fiorentino', Antonio di Jacopo, detto Antonio Gallo documentato a Firenze dal 1503 al 1527. 
all'identificazione del maestro va ritracciata, secondo la studiosa, nel tessuto di scambi tra Parigi e Lione. ${ }^{25}$

Giacché i pannelli non sono fronti di cassone, ma piuttosto "spalliere", rispetto al nome convenzionale attribuito da Zeri, è preferibile adottare la denominazione di Maestro delle "spalliere" Campana. Per quanto concerne il soggetto, Zeri ha rilevato che la più comune fonte delle Metamorfosi di Ovidio è rivista su Diodoro Siculo, confermando perciò il tono highbrow dei committenti. ${ }^{26}$ Invero, come vedremo dall'analisi iconografica, nella celebre serie di dipinti ad Avignone si individua un colore ovidiano che trapela da alcuni particolari ispirati non solo all'Ars Amatoria, le Heroides, le Metamorfosi, ma soprattutto, per intermediazione, ai commenti umanistici che corredano le opere dell'autore latino. ${ }^{27}$ Dettagli iconografici, indizi, parole nascoste, ci aprono dei sentieri e ci conducono alle fonti letterarie e alla committenza di queste opere così raffinate, risultato di un intreccio di fili derivati da glosse e scritte a margine del testo principale, una complessa tessitura di segni e concetti.

Il primo dipinto della serie (Fig. 5) raffigurante le nefaste origini del Minotauro mostra un tessuto narrativo molto articolato che si snoda dall'alto, a sinistra: il re cretese Minosse e la moglie Pasifae sacrificano un toro a Poseidone, ma non il migliore come richiesto dalla divinità. L'animale più bello, quello bianco con la stella dorata sulla fronte, si trova dietro Pasifae ed è stato risparmiato. Poseidone, per vendicarsi, fa innamorare la donna del toro bianco. Al centro del pannello, la moglie del re si trova dinanzi a Poseidone con l'attributo del tridente e gli indica il bianco toro. A sinistra, in primo piano, è raffigurato il palazzo di Minosse come indica il leone scolpito sulla rampa delle scale che ostenta uno scudo con il nome del re. Al balcone Pasifae osserva l'animale da lei amato, scende le scale e si precipita verso il toro a destra del dipinto. A seguito di una consultazione delle fonti, questo è il dettaglio che sembra maggiormente caratterizzare il contesto iconografico e tradire il riflesso di un passo preciso: Pasifae si prende cura del toro nutrendolo con erba fresca, come si rileva in Ars amatoria I, 289-304. ${ }^{28}$ L'animale raffigurato è contraddistinto sulla fronte da una stella che rimanda al poemetto ovidiano che descrive un toro bianco, chiazzato appena di nero in mezzo alle corna,

25. Seidel, 2015, p. 283.

26. Zeri, 1976 , p. 80.

27. Per un approfondimento della lettura iconografica dei pannelli di Avignone si veda Caciorgna, 2017.

28. Forte sub umbrosis nemorosae vallibus Idae / candidus, armenti gloria, taurus erat / signatus tenui media inter cornua nigro; / una fuit labes, cetera lactis erant. / Illum Cnosiadesque Cydoneaque iuvencae / Optarunt tergo sustinuisse suo. / Pasiphae fieri gaudebat adultera tauri; / invida formosas oderat illa boves. / Nota cano; non hoc, centum quae sustinet urbes, / quamvis sit mendax, Creta negare potest. / Ipsa novas frondes et prata tenerrima tauro / fertur inadsueta subsecuisse manu; / it comes armentis, nec ituram cura moratur / coniugis, et Minos a bove victus erat. / Quo tibi, Pasiphae, pretiosas sumere vestes? / Ille tuus nullas sentit adulter opes. 
signatus tenui media inter cornua nigro. Nel dipinto di Avignone la macchia dell'animale corrisponde in maniera precisa al testo sebbene il colore da nero diventi dorato. Interessanti risultano anche i versi in cui Ovidio insiste sull'attenzione dedicata da Pasifae al proprio aspetto per piacere al toro, ciò che si riflette nell'iconografia in cui la donna ostenta vesti preziose e si mette in mostra sul balcone del palazzo.

Il passo di Pasifae che nutre il toro è ripreso anche da Boccaccio in vari testi, fra i quali il Filocolo (IV 42) e non poteva essere altrimenti giacché i due protagonisti Florio e Biancofiore si innamorano leggendo proprio l'Ars amandi di Ovidio. Anche nell'Amorosa Visione (XXII, 28-33) Pasifae, con le proprie mani, taglia le fresche erbette dal prato fogliuto e le porge all'animale.

L'antefatto della storia, ovvero i precedenti dell'innamoramento di Pasifae, è narrato, ad esempio, fra le fonti antiche, in Apollodoro III, 1, tuttavia, non sembra questa la strada per l'esegesi iconografica, cioè richiamare un'altra fonte per la contaminatio. Si può seguire un altro percorso che ci guida alla lettura dei commenti ovidiani, in cui si riscontrano i vari elementi descrittivi rappresentati nel dipinto, come ad esempio quello pubblicato nel $1494 \mathrm{da}$ Bartolomeo Merula, umanista mantovano che contribuì alla diffusione dei classici: Enarrationes in Ovidium de arte amandi et de remedio amoris. ${ }^{29}$ Nella banda della pagina si legge l'annotazione marginale Pasiphaes fabula e l'esposizione della storia è narrata alla glossa candidus taurus:

Il CANDido TORo. Descrive il toro del quale Pasifae si infiammò. Raccontano le leggende che Pasifae, moglie di Minosse, poiché si voleva accoppiare con il toro, fu creata da Dedalo una statua in aspetto di vacca e che lei, postasi al suo interno e fattasi montare dal toro, appagò in tal modo la sua foia. Prima di allora raccontano che Minosse, come d'abitudine, ogni anno solesse sacrificare a Nettuno il toro più bello, ma disponendo quella volta di un toro più possente di tutti gli altri, gliene sacrificò uno inferiore. Per questo motivo Nettuno, irato con Minosse, infuse amore in Pasifae per quel toro, al quale, sottopostasi con l'inganno grazie all'arte di Dedalo, come si racconta nelle favole, generò il Minotauro dalla duplice natura, per modo che fosse toro nella parte superiore e uomo nell'altra: l'autore è Diodoro. ${ }^{30}$

29. IGI 7055. Sul Merula si veda Kristeller, 1967, pp. 277, 431; Perosa, 2000, pp. 575-576, nota 1.

30. Enarrationes in Ovidium de Arte Amandi et de Remedio amoris diligenter et accurate compositas a Bartholomaeo Merula Mantuano, impressit Venetiis vir solers et industrius Ioannes de Tridino alias Tacuinus, anno salutis 1494, b3v. Candidus taurus. Taurum describit cuius amore capta fuit Pasiphae. Tradunt fabulae Pasiphaen, Minois uxorem, cum taurum adamaret signum bovi simile a Daedalo factum eoque modo tauro suppositam desiderio potitam esse. Ante hoc tempus Minoem quotannis ferunt speciosissimum omnium taurum Neptuno solitum de more sacrificaret, quum ei taurus esset caeteris praestantior alium sacrificavit deteriorem. Qua ex re iratus Minoi Neptunus eius tauri amorem Pasiphae iniecit, cui arte Daedali supposita decantatum fabulis genuit Minotaurum natura duplici, ut qui usque ad humeros taurus caetera homo esset: auctor Diodorus. Più o meno lo stesso contenuto si ritrova nel commento alle Metamorfosi redatto da Raffaele Regio. 
Secondo una tecnica consueta, il commento rimanda peraltro a un'altra fonte che contamina il testo ovidiano prescelto, ossia Diodoro (IV, 77, 1-4), richiamato anche da Federico Zeri. D'altra parte il Merula aveva messo mano all'edizione del 1496 di Diodoro Siculo, già tradotto da Poggio Bracciolini. ${ }^{31}$ Proprio l'umanista sembra aver dunque operato nel suo commento la contaminazione Ovidio-Diodoro. Sulla sinistra del dipinto è raffigurato Dedalo, provvisto di un'ascia, mentre uccide una vacca di color marrone la cui pelle servirà per costruire il marchingegno che permetterà l'accoppiamento di Pasifae con il toro bianco. A destra, sullo sfondo, Pasifae appaga la sua foia entrando nella "macchina", mentre Poseidone, responsabile dell'accaduto, presiede alla scellerata unione. $\mathrm{Ci}$ aspetteremmo, tuttavia, la presenza di Dedalo piuttosto che quella di Poseidone. In conclusione, l'Ars amatoria e il commento relativo contengono elementi sufficienti a delineare il contenuto rappresentato dal pittore. Alcune spie, inoltre, come il gesto di nutrire il toro, rimandano proprio al testo di Ovidio. L'immagine di colore ovidiano è raffigurata a destra in maniera evidente, mentre altri particolari si ispirano alle glosse inserite dall'umanista a margine del testo antico. Anche il sentimento di piacere al bestiale amante da parte di Pasifae sul balcone in abiti eleganti è di matrice ovidiana, ripresa poi dalla cultura medievale come dimostra il testo di Boccaccio.

Nel pannello Minosse conquista Atene (Fig. 6) si osserva un corteo di soldati al seguito del re di Creta, raffigurato, come nella spalliera precedente, con una lunga barba bianca. Il suo nome è scritto nello stendardo rosso sorretto dal soldato sul cavallo grigio, al centro. I prigionieri vengono condotti a piedi con le mani legate, mentre sullo sfondo si assiste all'assedio di Atene, raffigurata come una città medievale. Si tratta della vendetta di Minosse per la morte del figlio Androgeo, ucciso dagli ateniesi durante le Panatenee, celebrazioni in onore di Atena che prevedevano una serie di competizioni atletiche. Il giovane aveva partecipato alle gare atletiche risultando vincitore, suscitando così le gelosie del re ateniese Egeo che lo aveva fatto uccidere. Minosse, quale rivalsa per vendicare la morte del figlio, chiese ad Atene un tributo annuo di sette giovani e altrettante giovinette da offrire in sacrificio al fine di nutrire il Minotauro. Seguendo il filo conduttore di Ovidio quale ispiratore dei pannelli Campana, osserviamo che l'episodio è ricordato nelle Metamorfosi (VII, 453458). Nell'edizione con il commento di Raffaele Regio, in relazione a questi versi è inserita una vignetta esplicativa con la rappresentazione in primo piano dell'uccisione di Androgeo e sullo sfondo Minosse a cavallo che va a vendicare la morte del figlio. La scelta di questo episodio, cui è dedicata una xilografia, testimonia l'importanza riconosciutagli dal pittore. Il commento al testo di Raffaele Regio, a margine del testo, offre una descrizione approfondita, con un rimando a Diodoro Siculo e Plutarco quali ispiratori della glossa. Si nota, in particolare, l'influsso della Biblioteca (IV, 60-61) che ha contaminato il testo ovidiano:

31. IGI 3454. Cfr. Perosa, 2000, p. 261, nota 1. 
Minosse prepara la guerra. Che Minosse, figlio di Giove e di Europa, fosse re di Creta è sin troppo noto perché io debba parlarne. Il figlio di questo, Androgeo, divenne famoso per una gara di atletica, nella quale, riportando la vittoria nella palestra di Atene, si attirò la massima invidia; per questo fu ucciso a tradimento dal re Egeo. Vedendo che non tornava, il padre Minosse mosse guerra agli Ateniesi, invocando su di loro la peste e la fame, per vendicare la morte di Androgeo: Plutarco e Diodoro. ${ }^{32}$

In questo secondo pannello della serie il colore ovidiano è trasmesso non solo dal testo e la sua glossa, ma anche grazie all'influsso dell'incisione relativa all'episodio di Androgeo, sebbene sia stato eliminato l'elemento più trucido dell'omicidio. La selezione di questo episodio evidenzia il ruolo primario conferito alla morte del figlio amato dal re e le conseguenze relative che ne scaturiscono sono rappresentate nel pannello successivo concernente l'impresa più eroica, l'uccisione del Minotauro.

Nella terza spalliera (Fig. 7), raffigurante Arianna e Fedra accolgono Teseo che sconfigge il Minotauro, la nave ateniese, simile a una caravella, sopraggiunge con i prigionieri ateniesi. Sul fronte della stessa sono disposti alcuni stemmi identificati, a partire dall'alto, come Feroni, dell'Antella, Salviati, Medici, Soderini, Malaspina, Serristori e Sassi. ${ }^{33}$ La studiosa di araldica Francesca Fumi Cambi Gado sostiene che il primo stemma sia in realtà da identificare in quello della famiglia Braccesi. ${ }^{34}$ In generale, la molteplicità delle famiglie rappresentate e il periodo in cui sono state dipinte le spalliere, ci conducono a un momento storico tra la fine della repubblica e il ritorno al potere dei Medici. Annamaria Bernacchioni rileva che all'epoca furono numerosi i matrimoni che legarono le famiglie i cui stemmi compaiono nel dipinto. Fra i più importanti si segnalano quello di Maria Soderini, che sposa nel 1511 di Lorenzo di Pierfrancesco dei Medici, del ramo dei Popolani. I Soderini si erano già legati ai Malaspina con il matrimonio tra Pier Soderini e Argentina, mentre, nel 1516, Giovanni delle Bande Nere si unì a Maria Salviati. ${ }^{35}$

Nel pannello Teseo è ormai sceso dalla nave dei prigionieri e raggiunge il palazzo di Minosse, raffigurato sullo sfondo, ove viene accolto da Arianna e Fedra. In primo piano, a sinistra, osserviamo ancora le due sorelle, con il

32. Habebis candide lector P. Ovidii Nasonis Metamorphosin castigatissimam cum Raphaelis Regii commentariis, k8r. Bella parat Minos. Minoem Iovis filium ex Europa regem fuisse Cretae notius est quam ut sit exponendum. Huic Androgeus filius fuit athletico certamene clarus, quo cum Athenis ex palestra victoriam reportasset, maximam in se invidiam concitavit quapropter insidiis Aegei Atheniensum regis occisi. Cum domum non rediret, Minos pater adversus Athenienses bellum movit, ipsis et pestilentiam et famem imprecatus, ut Androgei caedem ulcisceretur auctore. Plutar. et Diod. Anche Boccaccio prescelse a tutte le leggende riferibili a Minosse quella che ne testimoniava il grande amore paterno: nel Filocolo (II 12) e nell'Amorosa Visione (VIII, 40-42).

33. Comunicazione scritta di L. Jéquier, citata in Laclotte-Moench, 2005, p. 130.

34. Alla famiglia Braccesi apparteneva il celebre umanista Alessandro, autore di volgarizzamenti tra cui quello della Historia de duobus amantibus di Enea Silvio Piccolomini, capo della seconda cancelleria, ruolo ricoperto subito dopo da Niccolò Machiavelli.

35. Bernacchioni, 2011, pp. 118-119. 
medesimo abbigliamento, di fronte all'eroe in armatura ed elmo dalle eleganti piume bianche e rosse, i colori di Firenze. Arianna, che indossa un diadema, consegna il gomitolo a Teseo che ricompare al centro mentre raggiuge il labirinto. A destra, sullo sfondo, sono rappresentati alcuni antecedenti in cui il Minotauro, che compie stragi, viene trascinato al centro del labirinto. In primo piano, Teseo combatte il Minotauro nel labirinto e le due sorelle attendono all'ingresso, mentre a sinistra della costruzione circolare si prospetta il dettaglio conclusivo della narrazione, in cui Teseo conduce per mano Arianna e Fedra verso la nave in attesa per la partenza. Nelle fonti antiche, tuttavia - si vedano a esempio i contesti ovidiani, come l'VIII delle Metamorfosi (166-176) - l'impresa dell'uccisione del Minotauro si lega ad Arianna e Teseo, ma non a Fedra. Come rileva Rajna, né in Catullo né in Ovidio, incontriamo rivale alcuna a cui la povera Arianna si veda posposta; Teseo l'abbandona: ecco tutto. In seguito, per il tramite di commentatori che, volendo spiegare anche senza possedere le cognizioni necessarie, assai spesso confondono, al posto di Arianna subentrò Fedra: cosa naturalissima, poiché costei era sorella di Arianna, e più tardi la storia d'Ippolito la mostrava moglie di Teseo. ${ }^{36}$ L'intrusione di Fedra nel plot narrativo si trova per la prima volta nell'Amorosa Visione di Boccaccio, che introduce la fuga di Teseo con la sorella di Arianna (XXII, 4-24): ed è questa la prima espressione letteraria della nuova leggenda. Il dolore dell'eroina abbandonata risulta così accentuato. ${ }^{37}$ Anche nel Trionfo d'amore di Francesco Petrarca (vv. 116-117), "Teseo si staglia fra Arianna e Fedra, punito per aver preferito ad Arianna la sorella". ${ }^{38}$

Seguendo ancora la traccia ovidiana, tuttavia, è possibile individuare un altro percorso che ci conduce all'esegesi iconografica del dipinto. La via da seguire è di nuovo quella relativa ai commenti stampati a latere delle edizioni a stampa. Nel commento di Raffaele Regio alle Metamorfosi, l'autore rielabora, sulla scorta di Boccaccio o di commenti precedenti, la storia tratta da Ovidio aggiungendo la figura di Fedra nel tessuto narrativo, Ariadnem una cum Phaedra sorore abducens:

Teseo... partì di sua volontà per Creta, fu salvato dall'aiuto di Arianna, una figlia del re Minosse che se n'era innamorata. Infatti gli mostrò come uccidere il Minotauro, e, fissato un filo alla porta, come uscire dal Labirinto edificato da Dedalo. Perciò, ucciso il Minotauro, portando con sé Arianna insieme alla sorella Fedra, prese il largo verso l'isola di Dia dove Arianna, lasciata dall'ingrato, lamentando di essere stata abbandonata e invocando l'aiuto degli dei, piacque così tanto al padre Libero che la sposò. ${ }^{39}$

36. Rajna, 1876, p. 178.

37. Allo stesso modo, nel De casibus virorum illustrium, Boccaccio annuncia la fuga di Teseo con entrambe le figlie di Minosse (I, VII, 8).

38. Bettini, Romani 2015, p. 224.

39. Habebis candide lector P. Ovidii Nasonis Metamorphosin castigatissimam cum Raphaelis Regii commentariis, $17 r$. Theseus... sponte in Cretam profectus, Ariadnae Minois regis filiae amore illi captae auxilio servatus est. Nam quomodo et Minotaurum interficeret, monstravit, et filo ad ianuam fixo e Labyrintho a Daedalo extructo egrederetur, ostendit. Quare 
Significativa risulta anche l'incisione che illustra il libro ottavo delle Metamorfosi, in cui si osservano il labirinto a sinistra, al centro Teseo accompagnato da un soldato e, a destra, Fedra e Arianna, le due sorelle insieme, così come nel pannello di Avignone. ${ }^{40} \mathrm{Ma}$ un'altra spia che nel dipinto avignonese rimanda al commento ovidiano è quell'anello con il filo legato all'ingresso del labirinto per permettere meglio il suo dipanarsi all'interno della intricata costruzione: et filo ad ianuam fixo, come si legge nel commento. Dettagli nascosti, tracce che ci accompagnano nella lettura iconografica. Anche la chiosa a gnosis in ignotis del commento apposto dal Merula all'Ars Amatoria attesta la presenza di Fedra (v. 527):

La fanciulla di Cnosso vagava folle per spiagge sconosciute. Teseo, una volta sconfitto il Minotauro grazie all'aiuto di Arianna, ritornava ad Atene con lei e la sorella Fedra. Ad Arianna aveva promesso di prenderla in moglie, invece al figlio Ippolito avrebbe dato Fedra. Tuttavia, approdando a Dia, abbandonò Arianna addormentata. Ripartì invece con Fedra. Arianna poi, finalmente ridestatasi, riempiva di lamenti ogni dove. Ma Bacco, che per caso passava da quelle parti, prese la fanciulla come sua sposa. ${ }^{41}$

Il titulus a margine di questo commento, inoltre, enfatizza il connubio Arianna-Fedra: Thesei Ariadnae ac Phaedrae Fabula. In conclusione si denota una contaminazione tra i due commenti ovidiani, da una parte quello alle Metamorfosi con il "filo fissato alla porta" e la partenza di Teseo con Arianna e Fedra, dall'altro quello all'Ars amatoria in cui si trova un passaggio in più, Teseo che riparte con Fedra, ipsa cum Phaedra abiit.

Nella quarta spalliera raffigurante l'Abbandono di Arianna, sullo sfondo di una marina, si snodano gli ultimi momenti della storia di Arianna, della sorella Fedra, e di Teseo (Fig. 8). A destra, si osserva un re che si getta da una torre del castello lambito dal mare: cade con la sua corona e lo scettro. Si tratta di Egeo, padre di Teseo, morto per un tragico errore del figlio, che, vittorioso sul Minotauro, durante il viaggio di ritorno non ha cambiato le vele nere con quelle bianche. Al centro è raffigurata Arianna dormiente in un letto a baldacchino con la posa del braccio che le circonda la testa che si ispira a un gesto consolidato nella statuaria antica, basti ricordare la celebre Arianna dei Musei Vaticani. L'alcova, così preziosa, ci riconduce al carme 64 di Catullo e al suo

occiso Minotauro Ariadnem una cum Phaedra sorore abducens in Diam insulam primum enavigavit, ubi ab ingrato Ariadna relicta, cum se desertam quereretur opemque deorum invocaret, Libero patri ita placuit ut et eam sibi desponsarit.

40. Habebis candide lector P. Ovidii Nasonis Metamorphosin castigatissimam cum Raphaelis Regii commentariis, $17 r$.

41. Enarrarrationes in Ovidium de Arte Amandi et Remedio amoris diligenter et accurate compositas a Bartholomaeo Merula Mantuano, c1v. Gnosis IN IGNOTIs [amens errabat arenis]. Theseus, superato Minotauro Ariadnae auxilio, cum ea et sorore Phaedra Athenas redibat. Ariadnae promiserat se eam ducturum uxorem, Hyppolito vero filio Phaedram daturum. Quare, quum Diam appulisset, Ariadnam dormiente reliquit. Ipsa cum Phaedra abiit, tum demum experrecta Ariadna eiulatu omnia complebat. Sed quum forte Bacchus ea iter faceret puellam in coniugem duxit. 
carattere di Epitalamio. Nel 'cielo' del letto da cui si dipartono le cortine del baldacchino, sotto al quale Arianna dorme placidamente, si legge "I... MOLA I", elemento che appare particolarmente significativo nella lettura dell'opera. In primo piano, a sinistra, Teseo, secondo la versione dei testi di Boccaccio e del commento ovidiano, fugge con Fedra verso la nave. Sullo sfondo, a sinistra, il dettaglio rivelatore che tradisce l'ispirazione diretta alle Heroides di Ovidio: Arianna agita un candido velo appeso a un legno per farsi notare da Teseo che ha ormai preso il largo con la sua nave. Anche la raffigurazione del corteo di Bacco nella tavola della collezione Campana mostra numerose convergenze con alcuni versi ovidiani, in questo caso con l'Ars amatoria. ${ }^{42}$ L'eroina sarà invitata dalla divinità a dimenticare la perdita dell'amato e, come riferisce Lorenzo il Magnifico nel Trionfo di Bacco e Arianna, a godere pienamente della giovinezza "che si fugge tuttavia / chi vuol esser lieto, sia: di doman non c'è certezza". In effetti, il corteo raffigurato nella spalliera evoca una mascherata del Rinascimento sul ritmo dell'opera del Magnifico, forse proprio quella composta in occasione del Carnevale del 1490, o anche del corteo descritto da Poliziano nelle Stanze per una giostra.

Il colto ambiente mediceo risulta dunque significativo, come d'altra parte dimostra lo stemma della famiglia, insieme a quello di altre casate fiorentine, posto sull'imbarcazione di Teseo. Sembrerebbe essere dunque questa la traccia da seguire per la possibile identificazione della loro committenza. L'elemento che ci conduce a una destinazione più precisa potrebbe comparire sulla sommità del baldacchino sotto al quale dorme Arianna, ancora ignara dell'abbandono, in cui, come accennato, compare l'iscrizione "I... MOLA I", ovvero "Imola" ripetuta più volte tutto intorno, alla quale gli studiosi non sembrano aver mai dato rilievo. ${ }^{43}$ In realtà, acclaratane la pertinenza e l'autenticità, si tratterebbe di un importante dato. La legenda "I....MOLA I", potrebbe rimandare, in effetti, a Caterina Sforza, contessa di Imola e di Forlì. Figlia di Galeazzo Maria Sforza, cresciuta nell'ambiente colto milanese, andò in sposa a Girolamo Riario, signore di Imola. In terze nozze si legò a Giovanni di Pierfrancesco de' Medici, detto il Popolano, giunto alla corte di Caterina in missione diplomatica, esiliato insieme al fratello Lorenzo per l'ostilità con il ramo collaterale dei Medici, quello del Magnifico. La signora dello stato imolese-forlivese si sposò segretamente con Giovanni nel 1497 e, dalla loro unione, il 6 aprile dell'anno successivo, nacque un figlio Ludovico, in onore del Duca di Milano, "raro ed eccellente nel mestiere delle armi". ${ }^{4}$

Ludovico fu in seguito ribattezzato con il nome del padre, Giovanni, e detto delle Bande Nere, quando divenne celebre capitano di ventura. Giovanni, orfano di padre a cinque mesi, perse anche la madre a soli undici anni.

42. Ovidio, Ars amatoria I, 539-546.

43. Ringrazio Dominique Vingtain, conservatrice en chef del Musée du Petit Palais in Avignon e la restauratrice Mme Pomey per il loro prezioso aiuto nella lettura dell'iscrizione con la lampada di Wood. Da una loro prima indagine la scritta appare contemporanea all'opera d'arte.

44. Rossi, ed. 2002, p. 15. Cfr. Cardini, 2001, p. 161. 
Nel testamento la madre Caterina aveva nominato tutori del figlio Francesco Fortunati, canonico di San Lorenzo, e il banchiere Jacopo Salviati. Da Jacopo Salviati il piccolo Giovanni fu cresciuto e ne sposò la figlia Maria il 15 novembre 1516. Dal loro matrimonio nascerà Cosimo, capostipite della dinastia dei granduchi di Firenze. ${ }^{45}$

Al di là dell'iscrizione, sia che vi si possa leggere o meno "I... MOLA I", la data 1516 appare significativa giacché proprio in quel torno di anni sono datate le spalliere e gli emblemi Medici e Salviati compaiono fra quelli apposti sull'imbarcazione. Teseo è l'eroe che combatte contro il Minotauro, figura mitologica che potrebbe rinviare ai Borgia, nemici di Caterina. Cesare, il duca Valentino assediò nell'autunno del 1499 Imola e Caterina, dopo aver resistito all'assedio combattendo lei stessa, fu fatta prigioniera nel gennaio 1500. Prima di essere rinchiusa nelle segrete di Castel Sant'Angelo, Caterina inviò il piccolo Giovanni a Firenze, dalla famiglia d'origine. Liberata nel 1501, la madre raggiunse il figlio scampando agli agguati tesi da Cesare Borgia e visse gli ultimi otto anni di vita insieme con Giovanni a Firenze, in particolare nella dimora di Castello. Borgia, tra il 1499 e il 1503, mirava a costruirsi un principato nell'Italia centrale. ${ }^{46}$ Caterina nella villa di Castello, luogo assai dilettevole cercò di educare il figlio negli studi tenendogli di continuo appresso maestri, ma il giovane fiero di natura poco apprezzando le lettere, mostrò inclinazione fin dai primi anni piuttosto nel cavalcare, nuotare, esercitarsi in tutti quei modi che al soldato convengono. ${ }^{47}$ La Sforza morì a Firenze nel 1509 in una casa di via Larga, la cosiddetta 'Casa Vecchia' dei Medici a nord di palazzo Medici Riccardi. ${ }^{48}$ Tutori del figlio Giovanni undicenne, come accennato, furono nominati Francesco Fortunati, canonico di San Lorenzo, amico del padre, del ramo dei Popolani e, appunto, Jacopo Salviati. Nel 1516, quando si provvide alla divisione del patrimonio mediceo tra i cugini Pierfrancesco di Lorenzo minor e Giovanni di Giovanni, al figlio di Caterina furono assegnati i capolavori di Botticelli, le celebri "allegorie mitologiche", quali la Primavera, la Nascita di Venere e la Minerva con il centauro. ${ }^{49}$ Si trattava di spalliere,

45. Un'altra interpretazione che potremmo avanzare per "I...MOLA I" è che l'iscrizione rimandi a immolo e ai suoi derivati. In questa prospettiva andrebbe forse riferito ai giovinetti ateniesi sacrificati al Minotauro, liberati grazie a Teseo e il filo di Arianna; oppure ai sacrifici per Bacco che compare in questo dipinto.

46. Cardini, 2001, p. 161.

47. Rossi, ed. 2002, p. 16. Un rimando a Caterina, madre del probabile committente, nella spalliera presa in esame, oltreché dalla scritta "I....MOLA I" potrebbe essere costituito anche dalle corolle ripetute sulle cortine del baldacchino. Sono fiori di gelsomino e Caterina Sforza è ritratta come 'dama dei gelsomini' nel dipinto di Lorenzo di Credi conservato nella Pinacoteca Civica di Forlì. Seconda una recente ipotesi anche la Monna Lisa di Leonardo sarebbe da identificarsi con Caterina Sforza: Soest, 2011. In realtà l'emblema araldico dei Riario, ai quali Caterina si legò con il primo marito, è la rosa canina che la dama, nel ritratto, inserisce in mezzo ai gelsomini.

48. Carl, 1990, p. 40.

49. Cardini, 2001, p. 166. 
mobili dipinti di qualità eccelsa per stile e tematica trattati, così come lo sono i pannelli di Avignone.

Assai interessante risulta la schiera di stemmi sull'imbarcazione, qui più volte richiamata, forse per enfatizzare l'unione delle famiglie fiorentine. In particolare sappiamo che il matrimonio di Giovanni dalle Bande Nere con Maria Salviati segnò il ricongiungimento tra i due rami medicei: quello dei Popolani dal quale proveniva il marito di Caterina Sforza, Giovanni di Pierfrancesco, e l'altro principale, detto di Cafaggiolo, dal quale proveniva Maria. ${ }^{50}$

In questa prospettiva, Teseo, protagonista delle storie, che indossa l'elmo con le piume bianche e rosse, colori di Firenze, pare voler impersonare proprio Giovanni de' Medici. Cercando un sostegno nella letteratura contemporanea, Nicolò Machiavelli, nel Principe, più volte menziona Teseo divenuto "Principe", al pari di Mosè, Ciro e Romolo per propria virtù, e non per fortuna. ${ }^{51}$ L'eroe è ricordato da Machiavelli come il restauratore dell'indipendenza di Atene e come colui che era riuscito a raccogliere i dispersi Ateniesi. ${ }^{52}$ Teseo rappresenta dunque per Machiavelli l'exemplum degli antichi, così come Giovanni delle Bande Nere costituisce il suo ultimo eroe. ${ }^{53}$

Si noti infine come l'armatura indossata da Teseo, precisamente raffigurata dal maestro delle spalliere Campana, sia molto simile a quella funebre del condottiero che, dopo varie peregrinazioni, è oggi conservata nel Museo Stibbert di Firenze. ${ }^{54}$ L'associazione visiva all'armatura cinquecentesca dell'eroe antico, Teseo, che sin dall'adolescenza, come ricorda Plutarco (Vita di Teseo VI,2) mostrò coraggio, forza fisica e fermezza d'animo, testimonierebbe dunque la propensione per il mestiere delle armi di Giovanni, inusitatae virtutis dux. ${ }^{55}$

In conclusione, in questo breve saggio, l'arte del "tessere" è il fil rouge che lega gli antichi ai "moderni": dall'èkphrasis di Catullo intessuta nella coltre alla spalliera di Girolamo del Pacchia di tematica nuziale alle eroine intrecciate da artisti del Rinascimento, da Penelope che tesse il sudario di Laerte ad Arianna che aiutò l'amato con un filo, da Minerva maestra nell'arte della tessitura ai commentatori dei testi antichi, umanisti-artigiani che intessono chiose, contaminano fonti e creano nuovi "tessuti narrativi". Letterati spesso dimenticati, "artigiani del mondo antico", chiosatori anonimi, ma che hanno influito sull'iconografia dei dipinti del Quattro-Cinquecento sia attraverso le loro postille che servono a intessere il ductus narrativo sia come ideatori del programma consultati da committenti prestigiosi e attentamente seguiti da artisti in cerca di ispirazione.

50. Cardini, 2001, p. 172.

51. N. Machiavelli, Il Principe, VI.

52. Cioci, 1992, p. 263.

53. Ridolfi, 1969, p. 390.

54. Ciseri, 2001, p. 218. Sui resti dell'armatura: Scalini, 2001, pp. 223-229.

55. Dall'epitafio di Paolo Giovio inciso sulla lapide di sepoltura Giovanni: Ciseri, 2001, p. 214, fig. 10. 


\section{Bibliografia}

Bari 2017 = F. Bari, Arte fiamminga al servizio della Repubblica: le botteghe di arazzeria a Siena nel XV secolo, in "Bullettino Senese di Storia Patria", 124, 2017, pp. $116-162$.

Bec $1984=$ Ch. Bec, Les livres des Florentins (1414-1608), Firenze, 1984.

Bernacchioni 2011 = A. Bernacchioni, Il 'Gallo fiorentino': il Maestro dei cassoni Campana. Un pittore francese nella Firenze rinascimentale, in Benozzo Gozzoli e Cosimo Rosselli nelle terre di Castelfiorentino. Pittura devozionale in Valdelsa (Castelfiorentino, 10 maggio - 31 luglio 2011), Firenze, 2011, pp. 117-127.

Bettini, Romani 2015 = M. Bettini, S. Romani, Il mito di Arianna. Immagini e racconti dalla Grecia a oggi, Torino, 2015.

Boccaccio, ed. 1967: G. Boccaccio, De mulieribus claris, a cura di Vittorio Zaccaria, in Tutte le opere di Giovanni Boccaccio, X, a cura di Vittore Branca, Verona, Mondadori, 1967.

Caciorgna 2004 = M. Caciorgna, Fortuna delle Heroides nella cultura senese. La figura di Enone, in M. Caciorgna, Il Naufragio felice. Studi di filologia e storia della tradizione classica nella cultura letteraria e figurativa senese, Sarzana (La Spezia), 2004, pp. 91-139.

Caciorgna 2008 = M. Caciorgna, Da Ovidio a Domenico da Monticchiello. Presenza e connotazioni paradigmatiche delle Heroides nella cultura senese del Rinascimento, in Siena nel Rinascimento: l'ultimo secolo della Repubblica (Siena, 28-30 settembre 2003, Graduate College di Santa Chiara, Aula Magna), atti del convegno promosso dall'Università di Warwick e da quella di Siena, con la collaborazione del centro Warburg Italia, Siena, Accademia degli Intronati, 2008, pp. 37-70.

Caciorgna $2013=$ M. Caciorgna, Civitas Virginis. Immagini di Sapienza nel codice figurativo tra Rinascimento e Barocco, in L'Iconologia di Cesare Ripa. Fonti letterarie e figurative dall'antichità al Rinascimento, Atti del Convegno Internazionale di Studi (Certosa di Pontignano, 3-4 maggio 2012), a cura di Mino Gabriele, Cristina Galassi, Roberto Guerrini, Firenze, 2013, pp. 97-115.

Caciorgna 2015 = M. Caciorgna, Francesco di Giorgio Martini and workshop. Fragment of a spalliera with the Abduction of Helen of Troy: The Flight of Helen's Attendants, in Carl Brandon Strehlke, Machtelt Brüggen Israëls (a cura di), The Bernard and Mary Berenson Collection of European Paintings at I Tatti, Milano, 2015, pp. 267-272.

Caciorgna $2017=$ M. Caciorgna, Ariadne una cum Phaedra: colore ovidiano e commenti umanistici nelle spalliere della Collezione Campana ad Avignone: un possibile complesso decorativo per Giovanni delle Bande Nere, in "Icononographica", 16, 2017, pp. 126-145.

Caciorgna, Guerrini 2003 = M. Caciorgna, R. Guerrini, La Virtù figurata. Eroi ed eroine dell'antichità nell'arte senese tra Medioevo e Rinascimento, Siena, 2003.

Cardini 2001 = F. Cardini, Giovanni gentiluomo, in Giovanni delle Bande Nere, a cura di Mario Scalini, Cinisello Balsamo (Milano), 2001.

Carl 1990 = D. Carl, La Casa Vecchia dei Medici e il suo giardino, in Il Palazzo Medici Riccardi di Firenze, a cura di G. Cherubini, G. Fanelli, Firenze, 1990, pp. 38-43.

Catullo, ed. 1996 = Catullo, Poesie, trad. it. G. Chiarini, Milano 1996.

Cecchini 1962 = G. Cecchini, L'arazzeria senese, in Archivio storico italiano, 120, $149-77$. 
Cioci 1992 = F. Cioci, L'epigrafe sul boccale (un dono importante), in "Faenza", 78, 1992, pp. 257-274.

Ciseri = I. Ciseri, Gli ultimi giorni di Giovanni delle Bande Nere, le esequie e il ritorno del suo sepolcro a Firenze, in Giovanni delle Bande Nere, a cura di Mario Scalini, Firenze, 2001, pp. 203-221.

Fiumi, Tempesta $2000=$ F. Fiumi, G. Tempesta, Caterina Sforza, una donna del Cinquecento. Storia e Arte tra Medioevo e Rinascimento (Imola, 5 febbraio - 21 maggio 2000), Firenze, 2000, pp. 139-145.

France 1500 entre Moyen Age et Renaissance 2010 = France 1500 entre Moyen Age et Renaissance, direction scientifique d'Elisabeth Tabuiret-Delahaye, Geneviève BrescBautier et thierry Crépin-Lebland, Paris, 2010.

Kristeller 1967 = P. O. Kristeller, Iter Italicum, II, London, Warburg Institute, 1967.

Laclotte, E. Moench = M. Laclotte, E. Moench, Peinture italienne, Musée du Petit Palais, Avignon, Paris, 2005.

Perosa 2000 = A. Perosa, L'edizione veneta di Quintiliano coi commenti del Valla, di Pomponio Leto e di Sulpizio da Veroli, in Studi di filologia umanistica, III, Umanesimo italiano, a cura di P. Viti, Roma 2000.

Rajna 1876 = P. Rajna, Le fonti dell'Orlando furioso, Firenze, 1876.

Ridolfi 1969 = R. Ridolfi, Vita di Niccolò Machiavelli, Firenze, 1969.

Rigotti 2002 = F. Rigotti, Il filo del pensiero. Tessere, scrivere, pensare, Bologna, 2002.

Rossi, ed. 2002 = G. G. Rossi, Vita di Giovanni de’ Medici figlio di Caterina Sforza celebre capitano delle Bande Nere, note di M. Fabi, introduzione e note aggiuntive di R. Di Bari, Pavia, 2002 (ed. or. 1833).

Sanfilippo 2013 = M. Sanfilippo, Coniunx semper Ulixis ero. Penelope nell'arte e nella letteratura dall'antichità a Cesare Ripa, Lugano, 2013.

Sanminiatelli 1967 = D. Sanminiatelli, Domenico Beccafumi, Milano, 1967.

Scalini 2001 = M. Scalini, I resti dell'armatura funebre di Giovanni delle Bande Nere e alcuni oggetti a lui legati, in Giovanni delle Bande Nere, a cura di Mario Scalini, Firenze, 2001, pp. 222-229.

Seidel 2015 = C. Seidel, Un Français à Florence? Les cassoni Campana reconsidérés, in La France et l'Europe autour de 1500. Croisements et échanges artistiques, "Rencontres de l'Ecole du Louvre", Paris, Ecole du Louvre, 2015, pp. 275-287.

Soest 2011 = M. Soest, Caterina Sforza ist Mona Lisa, die Geschichte einer Entdeckung, Baden-Baden, 2011.

Sidonio Apollinare, ed. 1990 = Le nozze di Polemio e Araneola (Sidonio Apollinare, Carmina XIV-XV), introduzione, testo, traduzione e commento a cura di Giovanni Ravenna, Bologna, Pàtron Editore ,1990.

Zaggia, Ceriana 1996 = C. Zaggia, M. Ceriana, I manoscritti illustrati delle "Eroidi" ovidiane volgarizzate, Pisa, Scuola Normale Superiore, 1996.

Zeri 1976 = F. Zeri, Diari di lavoro 2. Torino, Einaudi, 1976. 


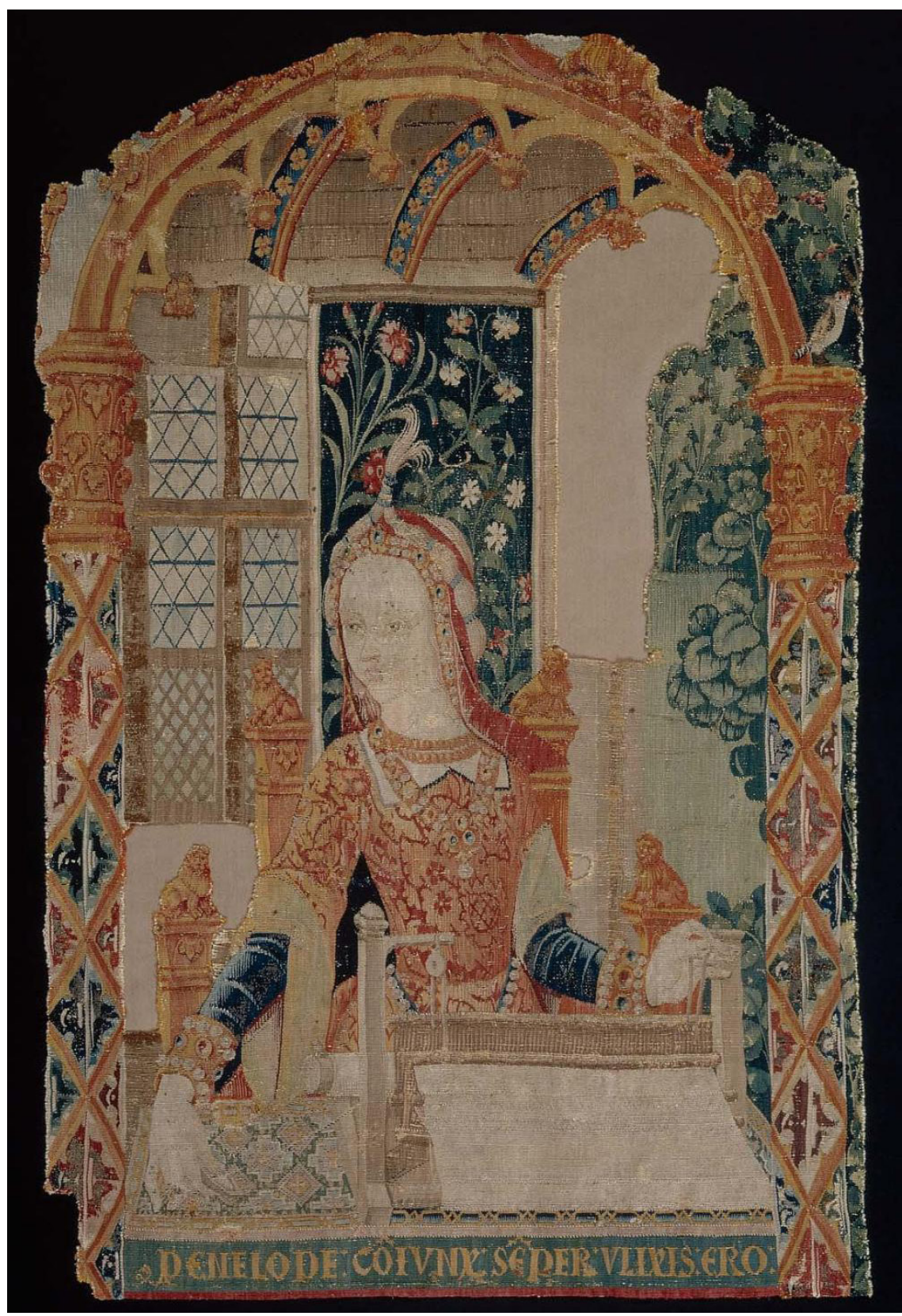

Fig. 1. Artista francese o franco-fiammingo, Penelope al telaio. Boston, Museum of Fine Arts. 


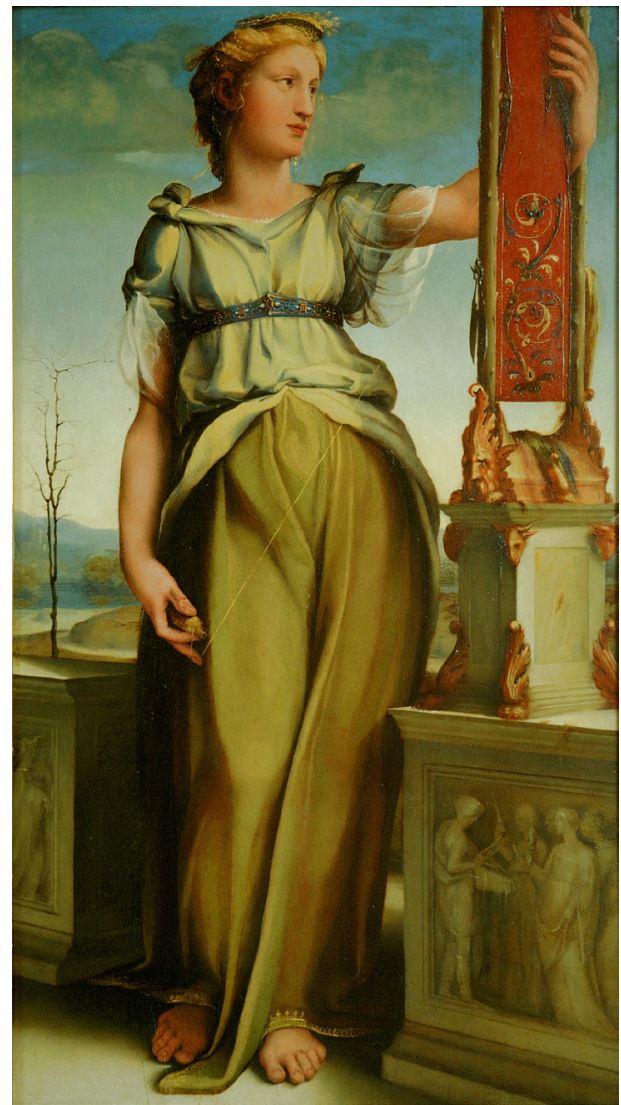

Fig. 2. Domenico Beccafumi, Minerva. Venezia, Pinacoteca Manfrediniana.

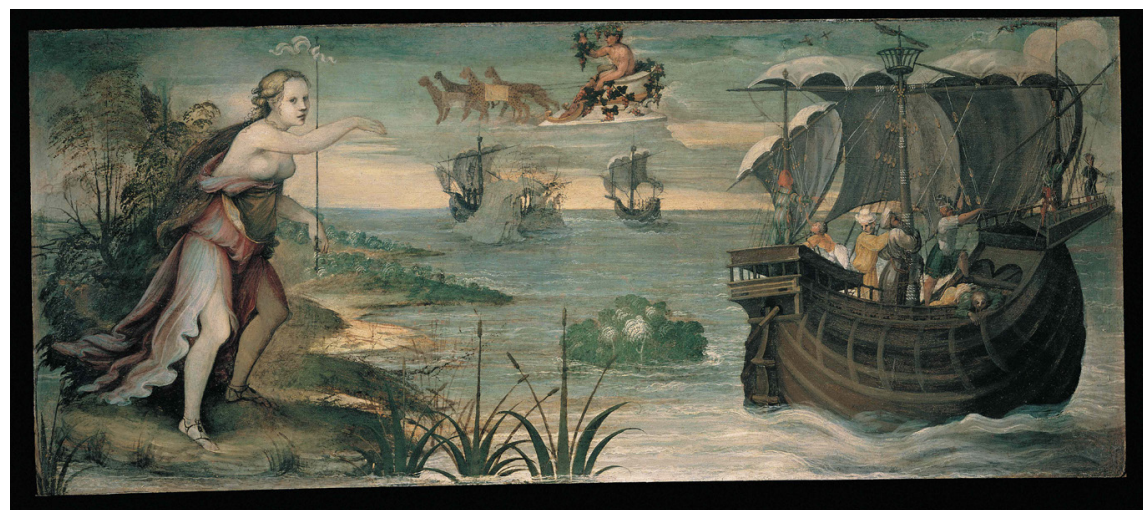

Fig. 3. Girolamo del Pacchia, Arianna abbandonata. Siena, Collezione Chigi Saracini. 


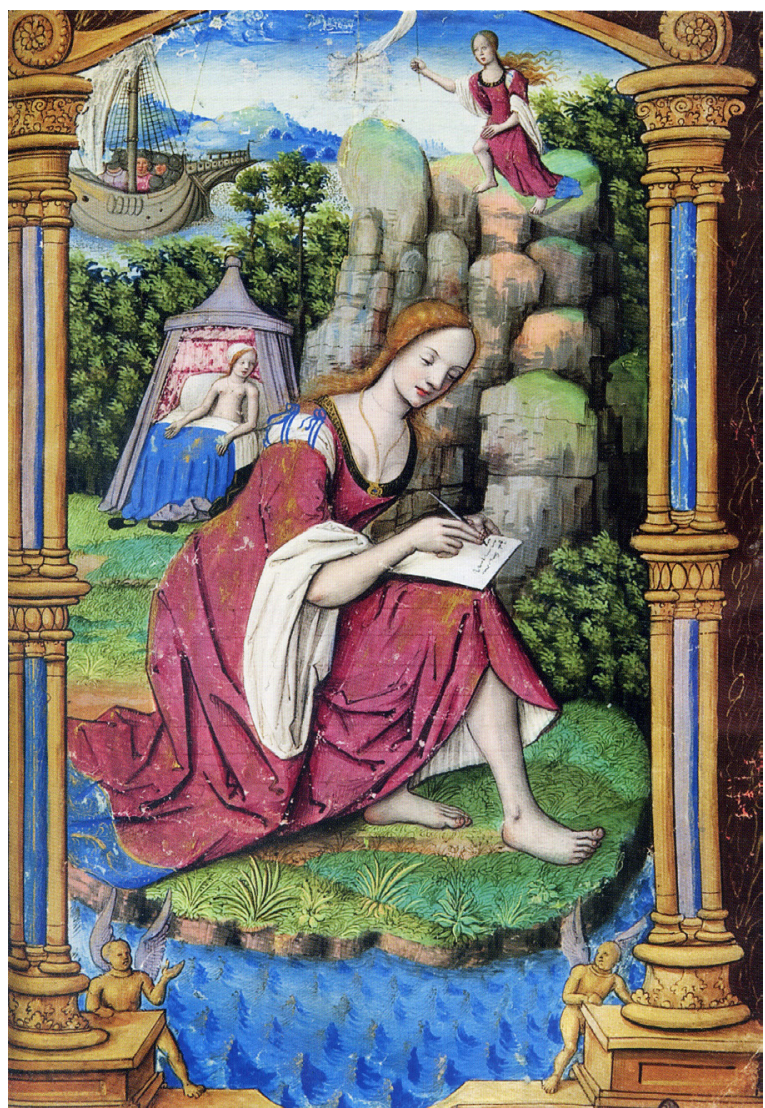

Fig. 4. Jean Pichore, Arianna abbandonata. Paris, Bibliothèque de l'Assemblée nationale, Ms. 1466.

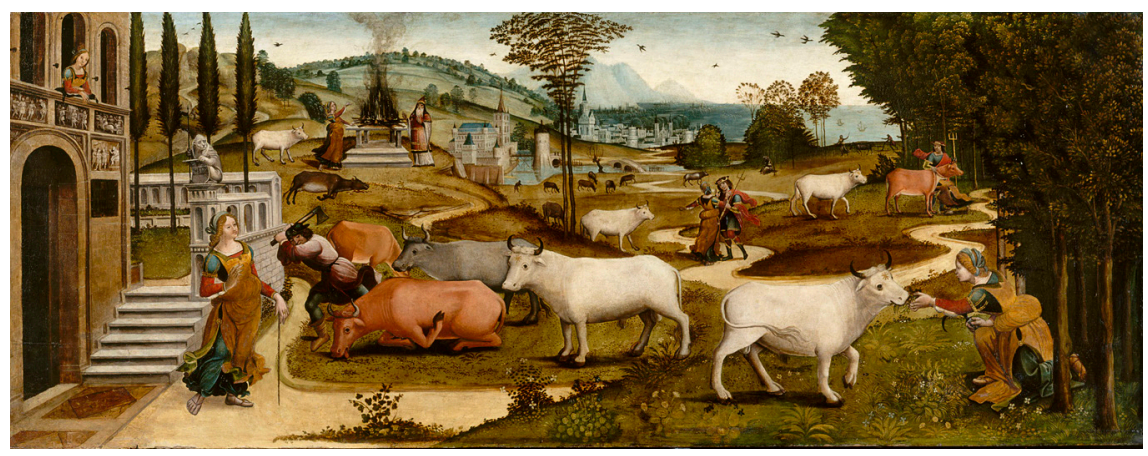

Fig. 5. Maestro delle spalliere Campana: Gli amori di Pasifae. Avignon, Musée du Petit Palais. 

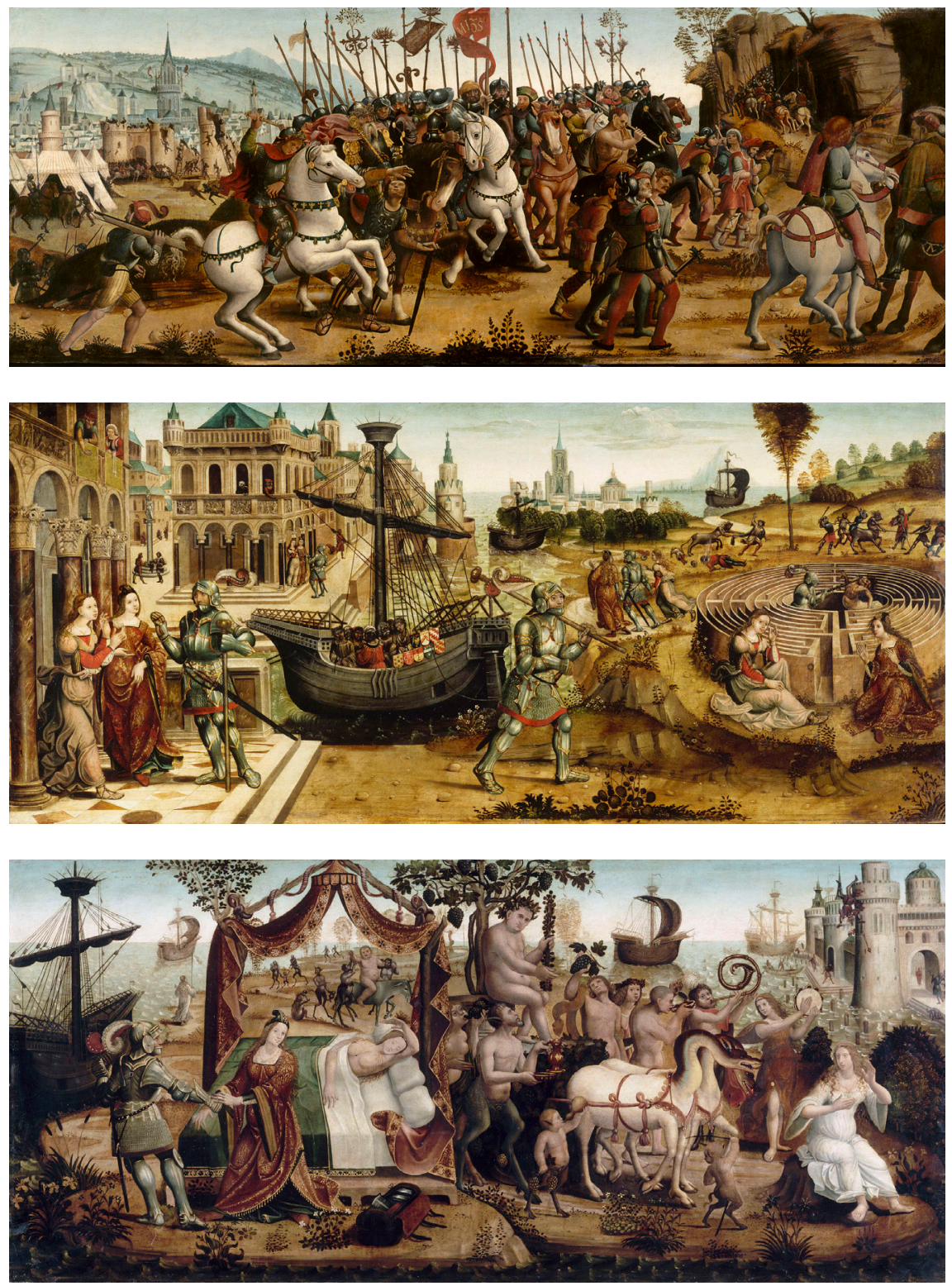

Figg. 6, 7, 8. Maestro delle spalliere Campana: Gli amori di Pasifae; Minosse conquista Atene; Arianna e Fedra accolgono Teseo che sconfigge il Minotauro; L'abbandono di Arianna. Avignon, Musée du Petit Palais. 
